\title{
Induction Treatment of Lupus Nephritis in Asian Countries: Is There a "Best" Therapy?
}

Jisoo Lee

Division of Rheumatology, Department of Internal Medicine, Ewha Womans University College of Medicine, Seoul, Korea

Lupus nephritis (LN) is a common and severe manifestation of systemic lupus erythematosus (SLE) putting a substantial burden on patients living with the disease. $\mathrm{LN}$ affects over half of the patients during the course of the disease [1]. Despite major improvements in treatment strategies over the last decade, LN still constitutes an important cause of renal failure with approximately $10 \%$ progressing to end-stage renal disease after 10 years $[2,3]$. Burden of LN on Asian SLE patients are more severe compared with other ethnic groups. In Asians, renal involvement is more prevalent and severe requiring research focusing on patients within this region [4-7]. Furthermore, genetic heterogeneity and differences in socio-economic status within Asian counties cause disparities in therapeutic responses and long-term outcome of $\mathrm{LN}$, but studies looking into the differences are scarce. A recent article by Choi et al. [8] published in Journal of Rheumatic Diseases provides data comparing therapeutic responses to different induction agents use for treatment of proliferative LN in ethnically homogeneous Korean patients.

In this retrospective study, the authors studied 39 patients who underwent induction therapy for treatment of active class III and IV proliferative LN. Twenty-three (59.0\%) patients were treated with intravenous cyclophosphamide (IVC) and 16 (41.0\%) treated with mycophenolate mofetil (MMF). Responses to both treatment regimens were compared at 6 and 12 months after the treatment. The analysis revealed that therapeutic responses were not significantly different between the 2 groups at both 6 and 12 months. Approximately half of the patients achieved complete response (CR) at 6 and 12 months despite the fact that IVC group had higher disease activity at baseline. In terms of safety, adverse events between the two groups were also not significantly different. Fifteen $(65.2 \%)$ of the patients who received IVC and 11 (68.8\%) of patients who received MMF experienced any adverse events. Major infection requiring hospitalization occurred only in MMF group $(n=3)$, but due to retrospective design of the study and small number of patients, the statistical significance could not be assured. The authors concluded that the IVC and MMF used for LN induction therapy are similar in terms of efficacy and safety in Korean patients with SLE.

Current standard of care regimens for proliferative (class III or IV) LN is induction therapy of high-dose corticosteroids combined with either high or low dose IVC or MMF followed by maintenance immunosuppression [9]. Most of the previous studies evaluating the efficacy of LN induction therapy in Asians were done in Chinese population since first data showing efficacy of novel therapeutic agent, MMF, came from the Hong Kong researchers [10]. In the prospective randomized trial in Chinese patients, they showed similar efficacy between MMF and oral cyclophosphamide, but MMF showed better tolerability [10]. Largest study comparing the efficacy of IVC and MMF as an induction agent for proliferative LN in Asians came from the Aspreva Lupus Management Study, an international multi-center study including 370 patients, of which 123 were Asians recruited from multiple centers in China and Malaysia [11]. They reported similar rates of response and toxicity between IVC and

Received : February 26, 2019, Revised : March 4, 2019, Accepted : March 4, 2019

Corresponding to : Jisoo Lee (iD http://orcid.org/0000-0001-6279-7025

Division of Rheumatology, Department of Internal Medicine, Ewha Womans University College of Medicine, 25 Magokdong-ro 2 gil, Gangseo-gu, Seoul 07985, Korea. E-mail : leejisoo@ewha.ac.kr 
MMF after 6 months of induction treatment.

Subsequently, reports followed from Malaysia, Japan, India, Nepal, and Korea comparing the efficacies of different induction treatment for LN. A randomized controlled trial in Malaysian patients showed no difference in efficacy and frequencies of adverse events between IVC and MMF for proliferative LN induction therapy [12]. In Japanese patients, 4 different induction therapies (high dose IVC, low dose IVC, tacrolimus, MMF) were compared in a retrospective study. They showed that $\mathrm{CR}$ rates and 3 years relapse free survivals were similar regardless of the induction regimen employed [13]. Two prospective studies were performed in Indian patients. One study compared low dose IVC with MMF showing comparable safety and efficacy as an induction treatment of less severe (exclusion of crescentic LN) class III, IV, and V LN [14]. Another study comparing 3 different induction agents (high dose CYC, low dose IVC, MMF) showed comparable rates of CR in all 3 treatment groups [15]. In a prospective study in Nepalese population, low dose MMF (maximum $1.5 \mathrm{~g} / \mathrm{d}$ ) showed similar efficacy and better safety profile compared with high dose IVC in class II, IV, and V LN [16]. In Korean population, 3 retrospective studies were published including the study by Choi et al. [8]. First study reported that IVC may be more efficacious in adverting-end stage renal disease and death than MMF [17]. Second study evaluated 6 and 12 month outcome between 3 induction regimens (high dose IVC vs. low dose IVC vs. MMF) in a relative large number of class III, IV, and V LN patients $(n=99)$. CR rates were shown to be similar between the 3 groups both at 6 and 12 months [18]. Lastly, the study by Choi et al. [8] compared efficacy and safety of high dose IVC and MMF as an induction therapy for proliferative LN showing comparability between the both treatments. Based on current evidences in Asian patients with LN, we can draw a conclusion that as in other ethnicities, various inductions agents show similar results.

The study by Choi et al. [8] is important in many ways. First, it fills in the knowledge gap regarding the variability in therapeutic responses and toxicities to different induction agents used for treatment of LN among ethnically different Asian populations. Second, it is powered by analyzing LN patients who have used standard dose of high dose IVC and MMF for comparison. Some of the study conclusions from Asian countries are weakened by including patients who had received variable and suboptimal doses of induction agents recommended for LN treatment $[14,17]$. Third, the study defines therapeutic responses to induction treatment in a confined group of patients with proliferative LN. Including membranous LN in evaluating therapeutic responses may act as a confounding factor since it has been shown that pathogenic mechanisms between proliferative and membranous LN are different [19].

The article contributed to current knowledge that there is no 'best' induction therapy for treatment of LN in Asians. This is good to know since both IVC and MMF appear to have similar efficacy and tolerability, induction using either IVC or MMF guarantee similar results in LN treatment. Rheumatologists within the region can select either agent for induction treatment based on their socioeconomic status and healthcare situations (i.e., cost and availability of drugs, and access to health care services and insurance).

\section{CONFLICT OF INTEREST}

No potential conflict of interest relevant to this article was reported.

\section{REFERENCES}

1. Tsokos GC. Systemic lupus erythematosus. N Engl J Med 2011;365:2110-21.

2. Vandepapeliere J, Aydin S, Cosyns JP, Depresseux G, Jadoul M, Houssiau FA. Prognosis of proliferative lupus nephritis subsets in the Louvain lupus nephritis inception Cohort. Lupus 2014;23:159-65.

3. Zhang L, Lee G, Liu X, Pascoe EM, Badve SV, Boudville NC, et al. Long-term outcomes of end-stage kidney disease for patients with lupus nephritis. Kidney Int 2016;89:1337-45.

4. Jakes RW, Bae SC, Louthrenoo W, Mok CC, Navarra SV, Kwon N. Systematic review of the epidemiology of systemic lupus erythematosus in the Asia-Pacific region: prevalence, incidence, clinical features, and mortality. Arthritis Care Res (Hoboken) 2012;64:159-68.

5. Seligman VA, Lum RF, Olson JL, Li H, Criswell LA. Demographic differences in the development of lupus nephritis: a retrospective analysis. Am J Med 2002;112:726-9.

6. Osio-Salido E, Manapat-Reyes H. Epidemiology of systemic lupus erythematosus in Asia. Lupus 2010;19:1365-73.

7. Yap DY, Tang CS, Ma MK, Lam MF, Chan TM. Survival analysis and causes of mortality in patients with lupus nephritis. Nephrol Dial Transplant 2012;27:3248-54.

8. Choi SE, Park DJ, Kang JH, Lee KE, Xu H, Lee JS, et al. Comparison of renal responses to cyclophosphamide and mycophenolate mofetil used as induction therapies in Korean patients with lupus nephritis. J Rheum Dis 2019; 26:57-65.

9. Hahn BH, McMahon MA, Wilkinson A, Wallace WD, Daikh DI, Fitzgerald JD, et al. American College of Rheumatology 
guidelines for screening, treatment, and management of lupus nephritis. Arthritis Care Res (Hoboken) 2012;64:797-808.

10. Chan TM, Li FK, Tang CS, Wong RW, Fang GX, Ji YL, et al. Efficacy of mycophenolate mofetil in patients with diffuse proliferative lupus nephritis. Hong Kong-Guangzhou Nephrology Study Group. N Engl J Med 2000;343:1156-62.

11. Appel GB, Contreras G, Dooley MA, Ginzler EM, Isenberg $\mathrm{D}$, Jayne $\mathrm{D}$, et al. Mycophenolate mofetil versus cyclophosphamide for induction treatment of lupus nephritis. J Am Soc Nephrol 2009;20:1103-12.

12. Ong LM, Hooi LS, Lim TO, Goh BL, Ahmad G, Ghazalli R, et al. Randomized controlled trial of pulse intravenous cyclophosphamide versus mycophenolate mofetil in the induction therapy of proliferative lupus nephritis. Nephrology (Carlton) 2005;10:504-10.

13. Hanaoka H, Kiyokawa T, Iida H, Ishimori K, Takakuwa $Y$, Okazaki T, et al. Comparison of renal response to four different induction therapies in Japanese patients with lupus nephritis class III or IV: a single-centre retrospective study. PLoS One 2017;12:e0175152.

14. Rathi M, Goyal A, Jaryal A, Sharma A, Gupta PK, Ramachandran $\mathrm{R}$, et al. Comparison of low-dose intra- venous cyclophosphamide with oral mycophenolate mofetil in the treatment of lupus nephritis. Kidney Int 2016;89: 235-42.

15. Sahay M, Saivani Y, Ismal K, Vali PS. Mycophenolate versus cyclophosphamide for lupus nephritis. Indian J Nephrol 2018;28:35-40.

16. Sedhain A, Hada R, Agrawal RK, Bhattarai GR, Baral A. Low dose mycophenolate mofetil versus cyclophosphamide in the induction therapy of lupus nephritis in Nepalese population: a randomized control trial. BMC Nephrol 2018;19:175.

17. Koo HS, Kim YC, Lee SW, Kim DK, Oh KH, Joo KW, et al. The effects of cyclophosphamide and mycophenolate on end-stage renal disease and death of lupus nephritis. Lupus 2011;20:1442-9.

18. Joo YB, Kang YM, Kim HA, Suh CH, Kim TJ, Park YW, et al. Outcome and predictors of renal survival in patients with lupus nephritis: Comparison between cyclophosphamide and mycophenolate mofetil. Int J Rheum Dis 2018;21:1031-9.

19. Rezende GM, Viana VS, Malheiros DM, Borba EF, Silva NA, Silva C, et al. Podocyte injury in pure membranous and proliferative lupus nephritis: distinct underlying mechanisms of proteinuria? Lupus 2014;23:255-62. 